\title{
A Simple Device to Obtain Reliable Foot Radiographs of Ambulatory and Non-Ambulatory Friedreich's Ataxia Patients
}

\author{
M. Billimoria', P. Allard ${ }^{1,2}$, J. Sibille ${ }^{5}$, J.P. Sirois ${ }^{6}$, M. Duhaime ${ }^{2,3}$ and G. Geoffroy ${ }^{2,5}$
}

ABSTRACT: A simple radiographic apparatus standardizing foot position and simulating weight-bearing effects in the feet is described in this paper. It provides an effective means of obtaining reliable radiographic data for both ambulatory and nonambulatory patients. This device is presently used in an on-going study on pes cavus in Friedreich's ataxia.

RÉSUMÉ: Un appareil radiographique simple à utiliser normalisant la position des pieds et simulant l'effet du poids du corps est décrit dans cet article. Il permet d'obtenir de manière efficace des données radiographiques fiables chez les patients ambulants où non. Cet appareil est présentement utilisé dans l'étude du pied creux chez les patients atteints d'ataxie de Friedreich.

Can. J. Neurol. Sci. 1984; 11:574-577

Radiographs are commonly utilized to confirm the presence and to assess the extent of foot deformities. Most often the clinician makes use of dorsiplantar (DP) and lateral (LAT) radiographs by marking on them reference lines and angles to document the state of the bony configuration and to quantitatively measure the observed deformity.

Standing radiographs are preferred since the weight-bearing attitude of the feet allows appraisal of the structural component of the deformity as opposed to the positional or reducible elements of the feet. Furthermore, in the case of a unilateral affliction, it allows comparison with the contra-lateral extremity being positioned and solicited in a similar manner (Gamble and Yale, 1975). Conventionally dorsiplantar radiographic views as described by Kreed (1979) are obtained when the foot is bearing the full weight of the body with its plantar aspect in contact with the X-ray cassette or with the foot assuming a similar position when the patient is sitting, semi-recumbent or supine. The $\mathrm{X}$-ray beam is usually centered over the second and third rays perpendicular to the film plate. To avoid the bony superimposition of the tarso-metatarsal joints the radiographic source is tilted by approximately $15^{\circ}$ towards the ankle joint. An alternative technique is to simply raise the forefoot by $15^{\circ}$ by inserting a wedge under the film cassette while keeping the X-ray source perpendicular to the table or ground level. Once the feet are positioned, the DP radiograph can then be taken.
To obtain a weight-bearing LAT radiograph of the feet, the patient is asked to stand on low leveled and radiotranslucent platform. Two film plates separated by a lead sheet are placed vertically between the feet and ankles while resting on the platform. The patient's feet are then positioned close together and in contact with the film packs. For each foot the LAT radiographs are taken individually and consecutively. With an horizontal tube projection, the X-ray beam is centered on the lateral aspect of the navicular-cuneiform region.

For a non-weight bearing LAT radiograph obtained in the sitting or semi-recumbent position, the patient's legs are placed in the "frog position" with the lateral aspect of the feet lying directly on the film cassette. Fig. 1 illustrates a typical set of DP and $\mathrm{LAT}$ radiographs of a Friedreich ataxia patient taken in the semi-recumbent position.

These current techniques however, present some limitations leading to inconsistant measurements. The radiographs are obtained with the patient assuming different positions: standing when possible or in supine lying. If more than one radiograph is required they are often viewed separately. The clinician usually quantifies a spatial foot deformity from its projection in a single plane namely that of the radiograph.

To illustrate the importance of proper foot positioning, Hlavac (1967) studied the effect of sequential changes of foot position from pronation to supination on $15 \mathrm{X}$-ray parameters using DP

From the School of Physical and Occupational Therapy, McGill University', the Paediatric Research Centre ${ }^{2}$, the Service of Orthopaedic Surgery ${ }^{3}$ and the Department of Neurology ${ }^{4}$. Sainte-Justine Hospital, Montreal, the Royal Ottawa Regional Rehabilitation Centre ${ }^{5}$ and the Department of Physical Education. University of Montreal ${ }^{6}$. Reprint requests for the complete supplement (Phase 3. Part 3) to: Prof. André Barbeau. Clinical Research Institute of Montreal, 110 Pine Avenue West, Montreal. Quebec, Canada H2W IR7. 


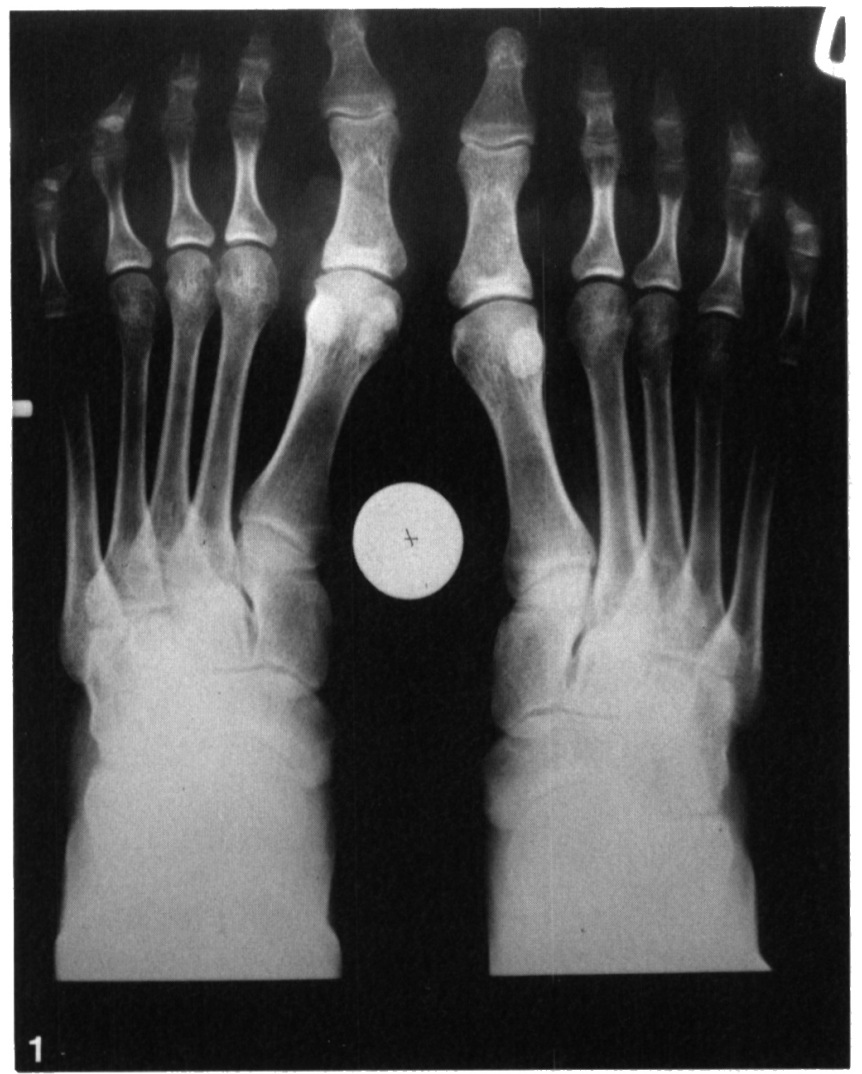

Figure 1-Foot Radiographs of a Friedreich's ataxia patient showing the (I) dorssiplantar and (2) lateral views.

and LAT radiographs of a foot of one normal 30 year old subject. He demonstrated that the values of all the parameters varied considerably. Three of them, namely the calcaneal inclination angle, the talocalcaneal angle and the talar declination angle showed a marked variation of $6^{\circ}, 9^{\circ}$ and $22^{\circ}$ respectively. With the foot pronated or supinated, there was a break in the cyma line in both DP and LAT views, whereas in the neutral position it was a smooth curve.

In an attempt to standardize foot radiographs Hammonds (1970) presents a device where the subject stands on it with his feet positioned in the angle of stance. The device allows a series of radiographs to be taken in the frontal, sagittal and transverse planes while the subject's position remains unchanged. The main disadvantage of this device is that its use is restricted to a subject capable of standing.

Others have developed radiographic devices simulating weightbearing for infants and patients unable to stand (Templeton et al., 1965; Ritchie and Keim, 1968). The values of the force exerted against the soles of the feet while a radiograph is taken is not mentioned in both studies.

This paper presents an apparatus to standardize foot position and to simulate weight-bearing effects in the feet for both ambulatory and non-ambulatory patients, allowing a possible comparison of the radiographic parameters obtained from the same patient or patients in different ambulatory stages, over a period of time.



\section{Apparatus}

The apparatus designed by Sibille et al. (1982) shown in Fig. 2 consists of a plexiglass frame (1) having a platform inclined at $15^{\circ}$ with respect to the horizontal on which the patient's feet rest. Two wires (2) which define an orthogonal $X$-ray reference system and eight spherical markers needed to correct for the magnification errors are imbedded in the platform. The straps (8) are used to restrain the feet. The X-ray cassettes are accommodated in slots (a) for DP and (b) for LAT views. The upper frame (3) houses a power screw (4), and handle (7), a compression spring (5) and a cushioned block (6), which are used to simulate weight-bearing effects in the feet. For mobility the entire apparatus is mounted on a wooden frame, resting on four castors. The combined height of the wooden frame and plexiglass platform is approximately as high as a regularly used clinical examination table.

\section{Procedure to Obtain Standardized Radiographs}

For taking standardized radiographs of the feet as shown in Fig. 3, the apparatus is placed at one end of an examination table. The patient lies supine on the table with his lips and knees flexed in a manner that allows his or her feet to rest on the plexiglass platform. The feet are maintained in neutral position by means of straps applied to the upper third of the shank and 


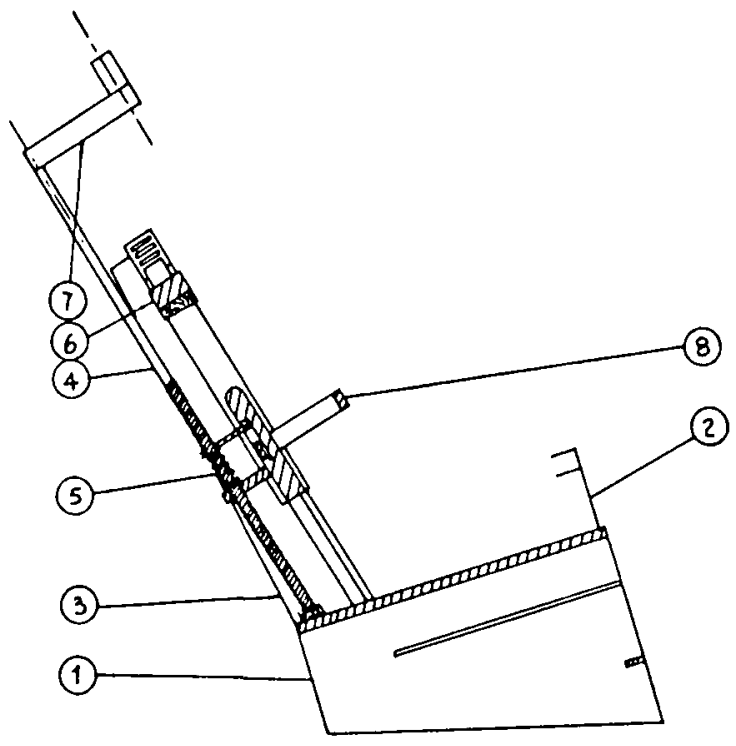

Figure 2 - Apparatus used to standardize foot radiographs while simulating weight-bearing effects.



Figure 3-Photograph of the device.

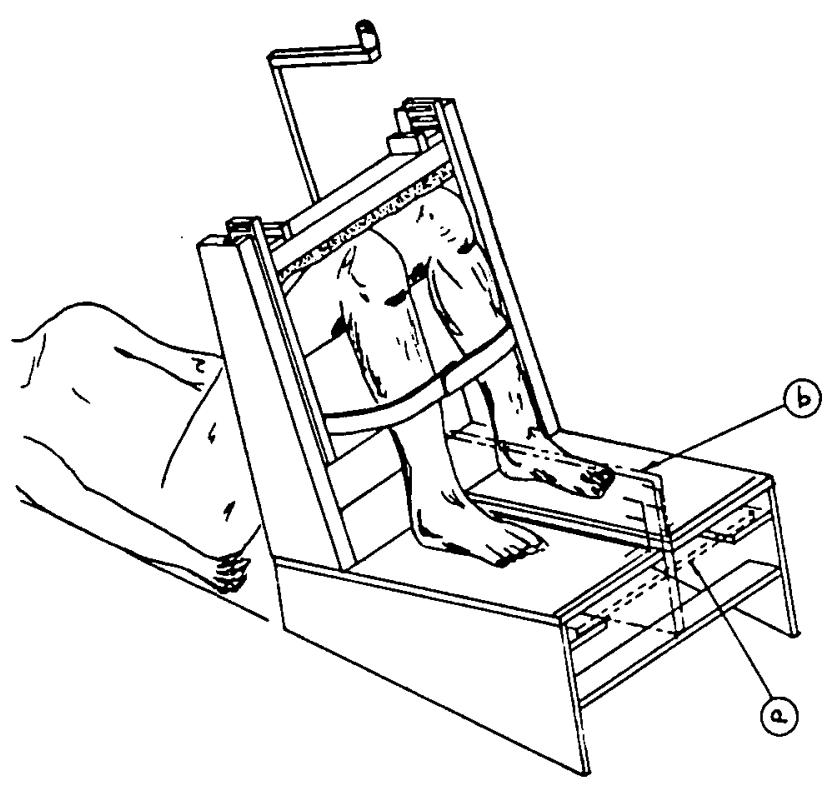

the ankles. The $15^{\circ}$ inclination minimizes bony superimposition on the radiograph.

To simulate weight-bearing effects in the feet, a force equivalent to the patient's body weight, is applied to his knees through the cushioned block using a handle to activate the power screw which in turn compresses a calibrated steel spring. Care is taken to ensure that the line of application of the force is parallel to the longitudinal axis of the shank. For the first of a pair of stereoradiographs, the X-ray source is appropriately adjusted at $750 \mathrm{~mm}$ above the cassette and positioned so that its beams are perpendicular to the second metatarsal. For the second radiograph it is moved laterally by $15^{\circ}$.

This apparatus ensures a standard foot position for all radiographs that are taken. The proposed method simulating weight-bearing effects in the feet provides comparable data for patients with a progressive deterioration of locomotion and non-weight-bearing patients. In addition, this apparatus can also be used to obtain three-dimensional data. This is achieved by the use of a pair of stereoradiographs shown in Fig. 4, calibrated by means of the spherical metallic markers and of a three-dimensional reconstruction technique which provides spatial as well as bi-dimensional parameters and their corresponding graphics.

This apparatus, called the Stool, is currently utilized to assess the evolutive pattern of pes cavus in Friedreich's ataxia at Sainte-Justine Hospital. 

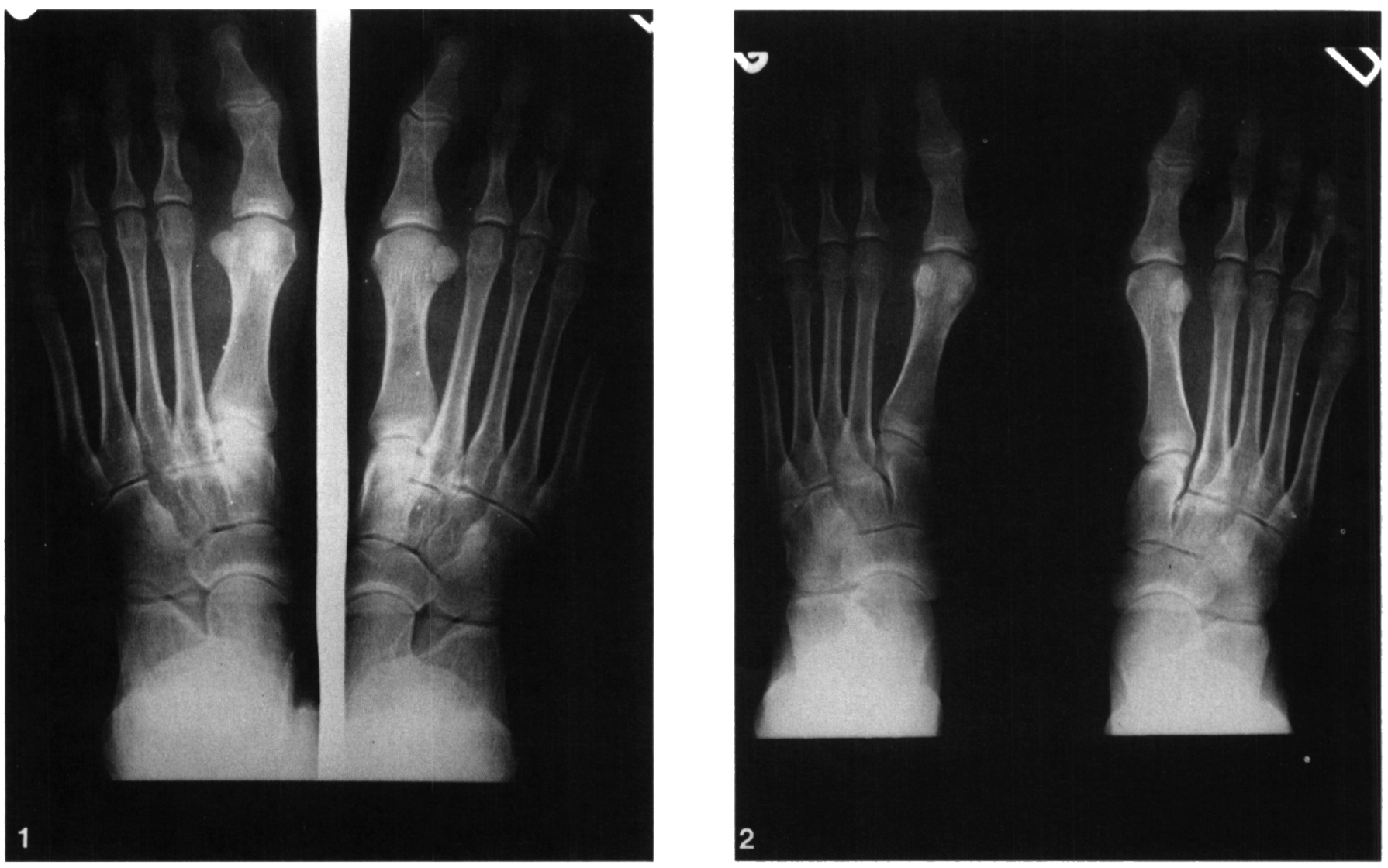

Figure 4 - A stereoradiographic image of the feet. The white dots on the photographs are the images of the radio-opaque spherical markers used in the calculation of three-dimensional parameters.

\section{ACKNOWLEDGEMENT}

This work was funded by l'Association Canadienne de l'Ataxie de Friedreich.

\section{REFERENCES}

Gamble FO, Yale I (1975). Clinical Foot Roentgenology, 2nd ed New York: RE Kreiger.

Hammonds CM (1970) Personal Interview. Clinical Foot Roentgenology 1975, 2nd ed, New York: RE Kreiger, pp 376-377.
Hlavac HF (1967) Difference in X-Ray Findings with Varied Positioning of the Foot. JAPA, 57: 465-467.

Kreed L (1979) Clark's Positioning in Radiography, William Heinemann Medical Books Ltd, 81-87.

Ritchie GW, Keim HA (1968) Major Foot Deformities, their Classification and X-ray Analyses. J Can Assoc Radiol 19: 155-166.

Sibille JP, Tremblay C, Thiry PS, Allard P (1982) Reference Apparatus for Normalized Bi-Planar Radiographs of the Foot. Journal of Biomechanics, 15: p 34.

Templeton AW, McAlister WH, Zim ID (1965) Standardization of Terminology and Evaluation of Osseous Relationships in Congenitally Abnormal Feet. Am J Roentgen. 93: 374-381. 